\title{
Randomized Assessment of the Safety and Efficacy of Intra-Arterial Infusion of Autologous Stem Cells in Subacute Ischemic Stroke
}

\author{
(D) V. Bhatia, DV. Gupta, DD. Khurana, DR.R. Sharma, and (D) N. Khandelwal
}

\begin{abstract}
BACKGROUND AND PURPOSE: Stroke is a debilitating illness for which treatment window is limited. Most patients present to the healthcare facility beyond that window. Autologous stem cells have shown some promise for this group of patients. This study was performed to evaluate the safety and the efficacy of intra-arterial infusion of bone marrow-derived mononuclear cells in patients with middle cerebral artery ischemic stroke.
\end{abstract}

MATERIALS AND METHODS: A prospective, randomized, open-label, blinded-end point study was performed from July 2015 to June 2016. Of 229 patients with acute stroke who presented to the hospital during this period, 20 patients who satisfied the inclusion/exclusion criteria were included and randomized into the control and intervention groups. Intra-arterial stem cell infusion into the ipsilateral MCA was performed in the patients in the intervention group at 8-15 days post-stroke ictus. Final analysis at 6 months was performed for primary (safety) and secondary outcomes (efficacy).

RESULTS: When we compared the primary end point of the study, no procedure-related mortality, complication, new infarct, or symptomatic intracranial hemorrhage was seen in the intervention group. When we compared the secondary end point of good clinical outcome, $8(80 \%)$ patients in the intervention group showed good clinical outcome (modified Rankin Scale score $<2$ ) with $4(40 \%)$ patients in the control group achieving this (95\% confidence interval for good outcome in patients with stem cell infusion, 49.03-94.3, and without stem cell infusion, $16.82-68.73 ; P=.068)$.

CONCLUSIONS: Intra-arterial infusion of stem cells can be carried out safely in the subacute stage of ischemic stroke. Improved clinical outcomes were observed with intra-arterial stem cell therapy; however, studies with larger cohorts are needed to validate the results.

ABBREVIATIONS: $\mathrm{BI}=$ Barthel index; $\mathrm{BMMNC}=$ bone marrow-derived mononuclear cells

$S^{t}$ troke is a leading cause of mortality and morbidity and is estimated to cause $>5$ million deaths per year throughout the world. Despite the recent advancement in therapeutic and rehabilitative strategies, stroke remains a major cause of financial burden on the health resources worldwide. Until recently, intravenous tissue plasminogen activator was the only approved therapy for acute stroke with a narrow window of 4.5 hours, and its current reach is to only up to $5 \%$ of the population. ${ }^{1,2}$

Recently, on the basis of multiple randomized trials, the Amer-

Received August 9, 2017; accepted after revision January 5, 2018.

From the Departments of Radiodiagnosis (V.B., V.G., N.K.), Neurology (D.K.), and Transfusion Medicine (R.R.S.), Postgraduate Institute of Medical Education and Research, Chandigarh, India.

Please address correspondence to Vivek Gupta, MD, Department of Radiodiagnosis, PGIMER, Sector-12, Chandigarh, India; e-mail: drvivekgupta.pgi@gmail.com

Indicates article with supplemental on-line tables.

Indicates article with supplemental on-line photo.

http://dx.doi.org/10.3174/ajnr.A5586 ican Heart Association and American Stroke Association have modified stroke treatment guidelines and criteria to include endovascular therapy with stent retrievers in acute stroke in a selected group of patients. ${ }^{2}$ However, only a limited number of patients with stroke reach the hospital in time for stroke revascularization therapies. Even in the dedicated stroke centers and institutions with aggressively organized stroke programs, only about $10 \%$ of patients with stroke can receive immediate treatment. ${ }^{3}$ Thus, most of these patients are eligible for only supportive treatment and rehabilitation. There are no definite pharmacologic or biologic interventions that can reverse impairment in these patients. ${ }^{4}$ Further research is required for treatment dedicated to reducing tissue-injury propagation and hastening clinical and functional recovery in these patients.

Stem cell infusion is a promising novel therapy for acute/subacute ischemic stroke. A few previous studies have examined the effect of bone marrow-derived mononuclear cells (BMMNC) in stroke using different routes of delivery. These studies have shown 
the safety and feasibility of stem cells in their respective patient cohorts, with good outcomes; but none were randomized. ${ }^{5-9}$ The aim of this research was to analyze in a randomized manner the safety and outcome of autologous BMMNC delivered directly into the ipsilateral middle cerebral artery in patients with ischemic MCA stroke of 8-15 days' duration.

\section{MATERIALS AND METHODS}

This prospective, randomized, open-label, blinded-end point study was carried out from July 2015 to June 2016 in a large tertiary care center in India. The study included patients presenting with acute MCA territory stroke. A total of 229 patients with acute stroke were admitted during this period. The initial management for acute stroke was performed in the patients who presented in and out of the therapeutic window according to the institutional protocol. Of these patients, 20 consecutive patients who fulfilled the inclusion and exclusion criteria were randomized into 2 groups (control and intervention groups) in accordance with the study protocol. Inclusion criteria were the following: age range of 20-80 years, symptoms and signs of clinically definite MCA stroke (0-14 days postictus), National Institutes of Health Stroke Scale score of $>7$, stroke clinically and on imaging conforming to the MCA territory, recanalization/patency of the involved M1 segment of the MCA on imaging, and patency of the carotid arteries for intra-arterial access of cerebral circulation. Exclusion criteria were the following: cerebral hemorrhage on CT/MR imaging, imaging evidence of M1 MCA segment complete occlusion, hemodynamic instability, known defect of clotting or platelet function, severe comorbidity precluding intra-arterial intervention, hepatic dysfunction, renal dysfunction, pregnancy, patients likely to be unavailable for follow-up, patients with evidence of chronic illness or advanced cancer, patients already dependent in activities of daily living before the present acute stroke (ie, prestroke mRS of $>3$ ), and refusal to give informed consent. The major reasons for exclusion included hemorrhagic stroke, scores outside the NIHSS criteria, occluded ipsilateral ICA or M1 MCA, and refusal to give consent.

The approvals were obtained from the ethics and stem cell ethics committees of the institution. A data safety-monitoring board was constituted, comprising 3 members, to report any adverse effects during the study. The data safety-monitoring board evaluated the occurrence of any adverse events, serious adverse events, and treatment-emergent adverse events during a meeting scheduled every 3 months.

\section{Clinical and Imaging Evaluation}

The patients were admitted and clinically evaluated by an experienced neurologist. History, detailed clinical examination, and clinical scoring based on the modified Rankin Scale scoring, National Institutes of Health Stroke Scale, and Barthel index (BI) were performed. Blood samples were sent for the following values: complete blood counts, blood urea, creatinine, lipid profile, blood sugar, and the serum electrolytes.

Written informed consent was obtained from the patient or their legal representative for the study. The same clinical and laboratory evaluations were also performed at day 1 postprocedure and at 1, 3, and 6 months in both groups. All patients who were included underwent a noncontrast head CT and brain MR imaging with an MR angiogram of the brain and neck. Patients were evaluated for infarct location, infarct volume, angiographic findings, the presence of any hemorrhage or new infarct, and the appearance of new lesions or any other findings. The MR imaging follow-up with similar imaging protocol, sequences, and parameters was performed in all patients at 1,3, and 6 months. An experienced neurologist and a neuroradiologist did the clinical evaluations and the interpretation of the images, respectively. Both were blinded to the initial clinical presentation and the nature of intervention.

\section{Bone Marrow Aspiration, Cell Separation, and Stem Cell Preparation}

The bone marrow aspiration was performed from the posterior superior iliac spine under aseptic conditions with the patient under local anesthesia. Each aspiration was transferred into a 350-mL collection bag containing citrate phosphate dextrose adenine, which was transferred to a bone marrow-processing laboratory for the further processing. The BMMNC were separated using a ficoll density gradient centrifugation procedure. Quality control of the final cell product was performed to determine the total number of cells, total BMMNC, total CD34+ cells, CD34+ cell percentage, sterility, and viability. Total nucleated cells and the mononuclear cells were counted using an automated hematology analyzer. CD34 cell enumeration was performed with an anti-CD34 antibody using the International Society of Hematotherapy and Graft Engineering guidelines. Viability was assessed using $0.4 \%$ Trypan Blue dye. The criterion set for the BMMNC dosage was a maximum of $5 \times 108$ cells to be infused.

\section{Intra-Arterial Stem Cell Infusion}

The right femoral artery access was through a $6 \mathrm{~F}$ arterial sheath. With a $5 \mathrm{~F}$ diagnostic catheter, ipsilateral internal carotid artery angiography was performed to ensure patency of the ICA and M1 segment of the middle cerebral artery to its bifurcation. Then, a $6 \mathrm{~F}$ Neuron guide catheter (Penumbra, Alameda, California) was placed in the ipsilateral ICA, and an Echelon 10 microcatheter (Covidien, Irvine, California) was navigated in the MCA and placed in the proximal segment (M1) of the MCA. Through the microcatheter, stem cell infusate was slowly injected in the MCA for 10 minutes. After the infusion, a check diagnostic run was performed to ensure patency of all the vessels and rule out any thromboembolic complication. Pre- and postprocedure heart rate, systolic and diastolic blood pressures, respiratory rate, and the presence of rash, urticaria, chills, and rigors or any other complication were noted.

\section{Evaluation of Results}

The data were evaluated for the primary and secondary outcomes according to the predetermined criteria. The primary end point (evaluation of safety) was clinically severe procedural complications (increase in the NIHSS score of $\geq 4$ points), symptomatic intracerebral hemorrhage, new ischemic lesions on imaging in the intervened territory, or death. The secondary end point (good clinical outcome) was defined as an mRS score of $0-1$ in patients 
Table 1: Demographics and baseline clinical features of the cohort

\begin{tabular}{lccc}
\hline & $\begin{array}{c}\text { Control } \\
\text { Group } \\
\text { (No.) (\%) }\end{array}$ & $\begin{array}{c}\text { Intervention } \\
\text { Group } \\
\text { (No.) (\%) }\end{array}$ & $\begin{array}{c}\boldsymbol{P} \\
\text { Value }\end{array}$ \\
\hline Age (mean) (yr) & $66 \pm 7.3$ & $57 \pm 12.2$ & .075 \\
Sex ratio (M/F) & $6: 4$ & $8: 2$ & .329 \\
Hemiparesis (L/R) & $5: 5$ & $6: 4$ & .653 \\
Thrombolysis & 0 & $1(10)$ & .305 \\
Hypertension & $5(50)$ & $5(50)$ & 1 \\
Diabetes mellitus & $6(60)$ & $7(70)$ & .63 \\
Dyslipidemia & $9(90)$ & $8(80)$ & .53 \\
Smoking & $1(10)$ & $5(50)$ & .051 \\
Atrial fibrillation & 0 & $2(20)$ & .13 \\
Coronary artery disease & $1(10)$ & $2(20)$ & .53 \\
Mean baseline NIHSS score & 10.5 & 10.6 & .94 \\
\hline
\end{tabular}

Note:- L indicates left; R, right.

with an NIHSS score of 8-14 at admission and an mRS score of $0-2$ in patients with an NIHSS score of $>14$ at admission.

\section{Statistical Analysis}

The Kolmogorov-Smirnov test was used for normality of distribution of the data. Continuous data were analyzed by a paired $t$ test, and discrete data, with $\chi^{2}$ tests. A value of $P<.05$ was significant.

\section{RESULTS}

\section{Baseline Demographic, Laboratory, and Stroke Indices}

There was no statistically significant difference between the 2 groups (intervention versus control) regarding the baseline demographic and clinical parameters (Table 1). Also, no statistically significant difference was seen between the 2 groups in relation to baseline stroke severity as assessed by the NIHSS $(P=.94)$ and the baseline clinical assessment with the modified Rankin Scale score $(P=.60)$ and the Barthel index $(P=.49)$. There was a trend toward higher baseline infarct size in the control group, but it was not statistically significant $(P=.065)$. On imaging, no statistically significant difference was seen in relation to infarct location, angiography findings, or the presence of hemorrhage or any other imaging findings at baseline and at 6 months.

Stem Cell Infusate Parameters and Comparison with Outcome. The mean poststroke day of intervention was day 10, the mean stem cell harvest volume was $118 \mathrm{~mL}$, the mean infusate volume was $5 \mathrm{~mL}$, and the mean total nucleated cells were $9.3 \times$ $10^{8}$. Mean mononuclear cells were $6.1 \times 10^{8}$, mean CD34 cells were $1.02 \times 10^{7}$, mean CD34 cell percentage was $1 \%$, and mean viability was $95 \%$ (On-line Table 1). There was no statistically significant difference between good outcome and poor outcome in relation to these parameters in the intervention group. There was no significant change in pre- and postprocedural heart rate, respiration rate, blood pressure, and temperature parameters in the intervention group. None of the patients had any allergic reaction such as urticaria, rash, chill, or rigor.

\section{Primary and Secondary Outcome Analysis}

Primary End Point Evaluation. No procedure-related complication was seen in any patient. No evidence of any immediate postprocedural or delayed complications related to the procedure or
Table 2: Comparison of primary and secondary outcomes between the 2 groups

\begin{tabular}{lccc}
\hline \multicolumn{1}{c}{ Parameter } & $\begin{array}{c}\text { Control Group } \\
(\boldsymbol{n}=\mathbf{1 0})\end{array}$ & $\begin{array}{c}\text { Intervention } \\
\text { Group }(\boldsymbol{n}=\mathbf{1 0})\end{array}$ & $\boldsymbol{P}$ Value \\
\hline Mortality & 2 & 1 & .53 \\
Complications & 0 & 0 & \\
New infarct & 0 & 1 & .305 \\
SICH & 0 & 0 & \\
Good outcome & 4 & 8 & .068 \\
\hline
\end{tabular}

Note:- SICH indicates symptomatic intracerebral hemorrhage.

the infused stem cells was seen. None of the patients in either group had episodes of symptomatic intracerebral hemorrhage immediately after the procedure or at follow-up. No fresh infarct in the involved hemisphere was seen on the follow-up imaging. One patient in the intervention group had an episode of infarction on the contralateral side 2.5 months after the procedure due to a cardioembolic phenomenon (Table 2).

None of the patients in either group had any neoplasms. Two of the patients in the control group died within 1 month of stroke. These patients had initial NIHSS scores of 15 and 18, respectively. Both patients had high initial stroke severity, and the probable cause of death was cardiac arrest. One patient in the intervention group (mentioned above) who had a contralateral stroke at 2.5 months died a month later. This patient had an initial baseline NIHSS score of 11, which had improved to 4 at discharge and up to 1 at the 1-month follow-up. Stroke in the contralateral MCA territory resulted in an NIHSS score of 22 at 3 months, which ultimately led to his death.

Secondary End Point Evaluation. Secondary end point evaluation for clinical improvement was based on the initial NIHSS score and the mRS score at 6 months. There were 8 (80\%) patients in the intervention group and $4(40 \%)$ in the control group who achieved good outcome $(P=.068$; odds ratio $=6$; $95 \%$ CI odds ratio, $0.81-44.31)$. Seventeen patients had a baseline NIHSS score of $8-14$. Of these, 12 patients, including 8 intervention cases and 4 controls, were regarded as having achieved a good clinical outcome (mRS of $0-1$ ) at 6 months (Figs 1 and 2). Three patients in the control group had an NIHSS score of $>14$. All of them had poor outcome at 6-month follow-up because an mRS score of $<2$ could not be achieved. Both groups showed improvement in the mRS during a 6-month follow-up; however, intragroup analysis revealed that this improvement was statistically significant only in the intervention group $(P=.009)$. There was a significant difference seen in the $\mathrm{BI}$ in the intervention group at 6-month follow-up compared with baseline $(P=.004)$. No statistically significant differences were seen in the control group (On-line Figure).

\section{DISCUSSION}

Ischemic stroke is one of the leading causes of morbidity and mortality worldwide because it leads to irreversible damage to the neuroglial tissue, resulting in functional deficits and chronic sequelae. The only therapy that is FDA-approved for acute stroke is intravenous $\mathrm{PA}$, which has the limitations of a narrow window (up to 4.5 hours for anterior circulation) and potential hemorrhagic complications. Therefore, it is currently benefiting a limited number of patients with stroke. ${ }^{10}$ Recently, the American Heart Association and the American Stroke Association have 


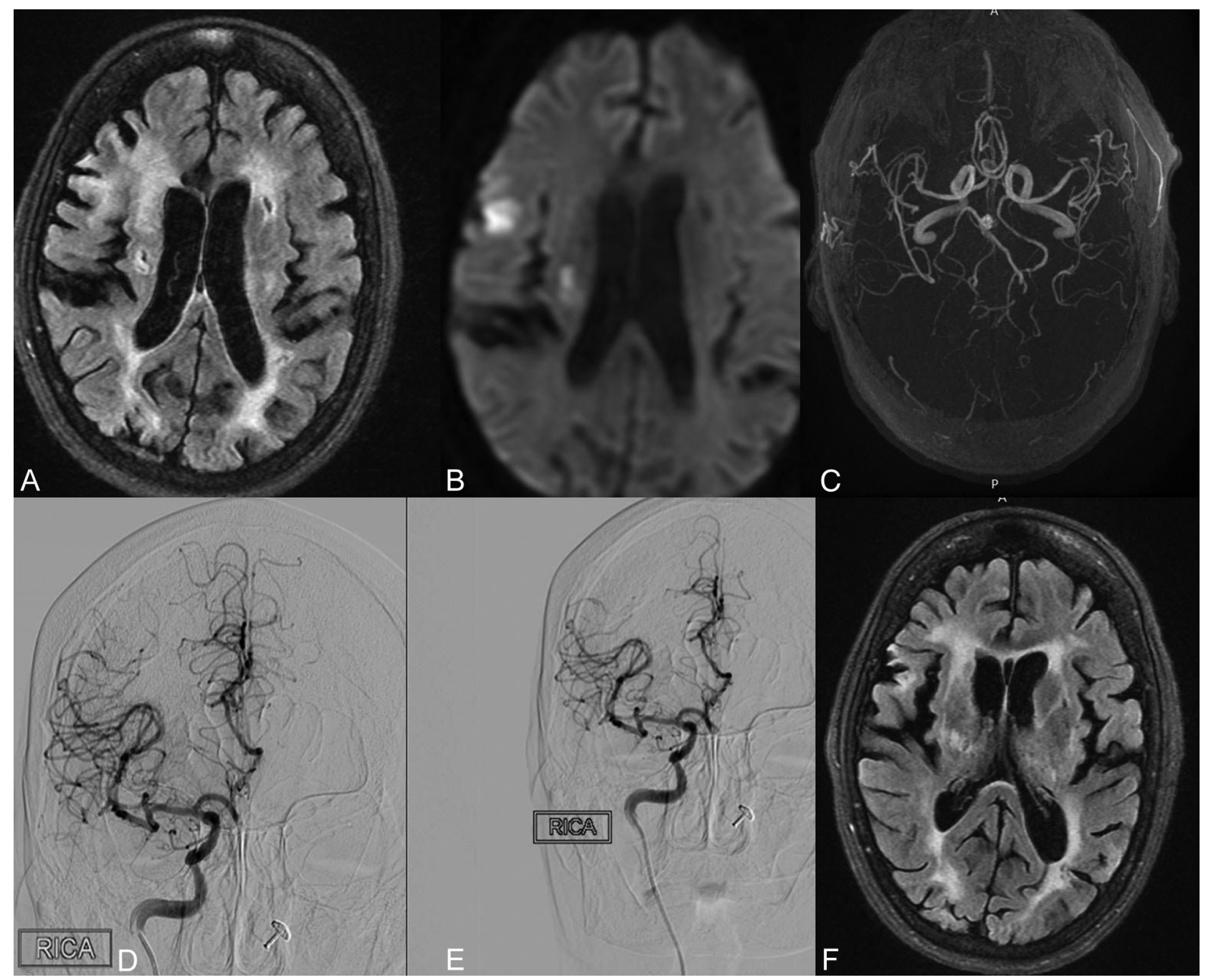

FIG 1. A 70-year-old man with left hemiparesis and a baseline NIHSS score of 10 (intervention group). Axial FLAIR image (A) shows an infarct in the right periventricular and posterior limb of the internal capsule, showing diffusion restriction on diffusion-weighted image (B). MR angiogram shows no evidence of any major branch occlusion (C). Preprocedural right ICA diagnostic run (D) shows normal opacification of the MCA branches. Post-stem cell infusion right ICA run $(E)$ shows similar findings, with normal opacification of all the branches. Axial FLAIR MR imaging at 6-month follow-up shows a reduction in infarct size. This patient had an mRS score of 1 at 6-month follow-up, and his outcome was considered good.

modified their acute stroke treatment guidelines to include mechanical thrombectomy as a first-line treatment for selected patients with large and proximal artery occlusions. ${ }^{2}$ However, a large subset of patients with stroke are either not able to meet these criteria or, due to time or cost constrains, are not able to take advantage of this form of treatment. Therefore, a large number of patients with acute stroke will either not receive any definitive treatment or, even after the treatment, may be left with residual neurologic deficits.

Stem cell therapy is a relatively novel approach in the treatment of patients with stroke, with the fundamental hypothesis coming from the observation that certain brain areas such as the dentate nucleus of the hippocampus and the subventricular zone are capable of regeneration and neurogenesis. ${ }^{11}$ Patients for stem cell therapy can be selected on the basis of the neuroprotective outcome for acute stroke or neuroreparative outcome for damaged brain tissue to promote neural tissue endogenous repair. Various mechanisms, which potentially produce benefits in pa- tients with stroke, include reduced apoptosis and inflammation, promotion of angiogenesis and neurogenesis, promotion of neural plasticity, and formation of neural circuitry. ${ }^{11,12}$

The different methods for transplantation of stem cells in patients with stroke include direct intracranial, arterial, and venous routes. ${ }^{13}$ Intra-arterial transplantation in the affected territory is an invasive approach, but it can directly implant these cells in the affected territory with less risk than direct stereotactic implantation and has the advantage of more dose deployed compared with the intravenous route. ${ }^{13,14}$

In our study, we obtained autologous BMMNC from the patients with stroke on the day of transplantation, which were then transplanted through an intra-arterial route directly into the ipsilateral MCA. We compared our results with age- and sexmatched controls.

Our study was a prospective, randomized, open-label, blindedend point study involving 20 patients with acute ischemic stroke, 10 of whom received intra-arterial stem cells (intervention group) 


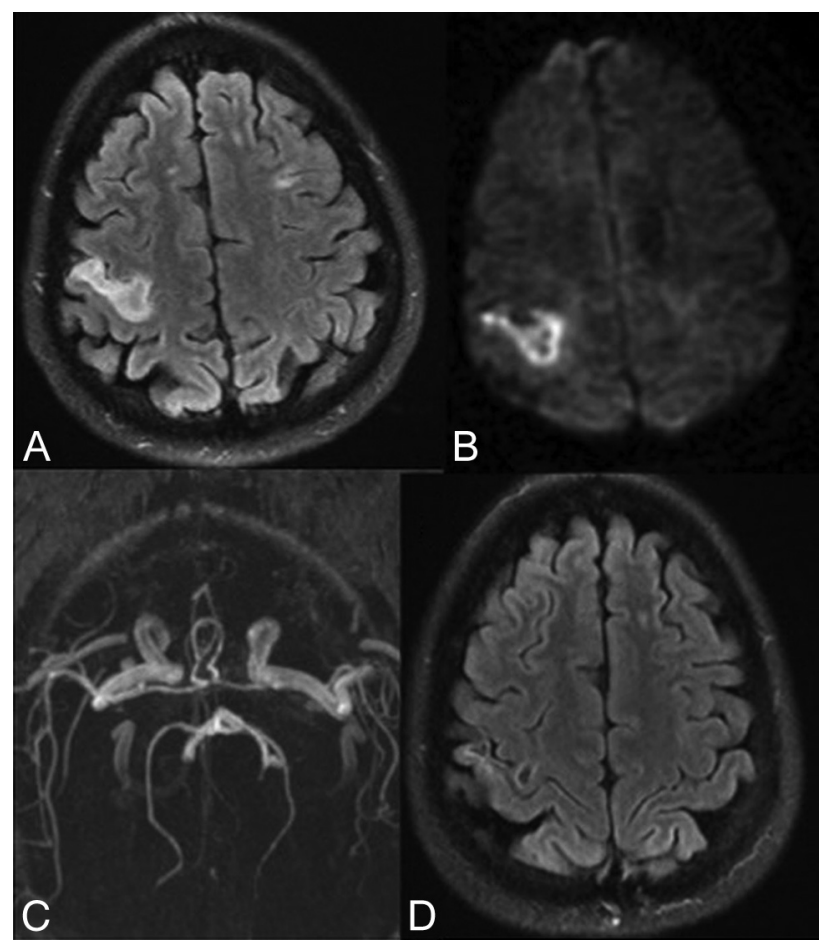

FIG 2. A 50-year-old man with right hemiparesis and an NIHSS score of 7 (intervention group). Axial FLAIR image ( $A$ ) shows an infarct in the right cortical subcortical location showing diffusion restriction on DWI (B). MR angiogram shows no evidence of any major branch occlusion. Axial FLAIR MR imaging at 6-month follow-up shows a reduction in infarct size. This patient had an mRS score of zero at 6-month follow-up, and his outcome was considered good.

with the rest acting as controls. Previous studies evaluating intraarterial stem cell therapy in acute stroke either did not have controls or lacked randomization. Also, the results were not evaluated in a blinded fashion. ${ }^{5-9,14}$ The present study has tried to remove the inherent bias by using the prospective, randomized, openlabel, blinded-end point study design.

The mean age in our study population was 61.7 years, with a majority of male patients $(n=14,70 \%)$. Eleven (55\%) patients had left-sided MCA infarct. There was no statistically significant difference between the 2 groups in relation to the age and laboratory values such as hemoglobin, total leukocyte count, platelets, international normalized ratio, urea, creatinine, blood sugar, electrolytes, total cholesterol, and so forth. Both groups received similar stroke care, including pharmacotherapeutics, physiotherapy, and rehabilitation.

Stem cell numbers and viability parameters in our study group were comparable with those in previous studies. ${ }^{5,9,14}$

We performed the stem cell implantation between 8 and 15 days after stroke. The mean poststroke day of intervention was 10 days in the present study. Previous studies using the intra-arterial route have demonstrated the safety of the procedure from 3 to 9 days. ${ }^{5,9,14}$ Increasing the time for stem cell implantation beyond 1 week of acute stroke as in the present study enables more patients with stroke to be included for the therapeutic benefit of stem cell infusion if proved. The comparison of parameters between the current study and the previous studies is given in On-line Table 2.

The primary end point of the study was the evaluation of the safety of the procedure, which was assessed by reviewing the mor- tality, symptomatic intracranial hemorrhage, presence of new infarcts/lesions, or any other complications. No intracranial hemorrhage was found in either group on follow-up. These data are consistent with previous studies that have reported the safety of transplanted stem cells in patients with stroke..$^{5-9,14}$ During the course of the study, 1 patient in the stem cell group died at 3.5 months follow-up due to the occurrence of a contralateral MCA infarct. This patient was treated for right-sided MCA infarct with intra-arterial stem cells. At 1-month follow-up, this patient had shown improvement with an mRS score of 2 (the NIHSS score at admission was 13 , and at discharge, it was 4). This patient had undergone an operation for atrial septal defect 20 years earlier, and the cause of stroke was probably cardioembolic due to persistent atrial fibrillation. The cause of the new stroke at 2.5 months in this case was again probably cardioembolic, resulting in an occluded left MCA. He was managed at this time with intra-arterial thrombectomy; however, the MCA could not be recanalized and the patient developed infarct and deteriorated. At 3-month follow-up, this patient had an mRS score 5. As in our study, Savitz et $\mathrm{al}^{8}$ also found 1 mortality due to pulmonary embolism at 40 days in their study population of 10 patients. The authors concluded that this mortality was not procedural-related because the patient was at high risk of developing deep venous thrombosis due to prolonged limb immobilization and this was the likely source of pulmonary embolism. They reasoned that previous studies have reported that the injected mononuclear cells die within a week of administration and therefore cannot account for the reported episode of pulmonary embolism approximately 1 month after the procedure in their study. ${ }^{15}$ Likewise, our patient also had a stroke after 2 and a half months, likely due to atrial fibrillation and not related to the intervention.

There were 2 mortalities in the control group within 1 month of stroke onset. Both had initial high stroke severity with NIHSS scores of 15 and 18, respectively. A previous study with a control population did not find any mortality in the control group. ${ }^{5}$ This difference is likely because of the higher NIHSS scores of these patients at presentation and subsequent deterioration. No other patient developed any other infarct in the intervened side or neoplastic lesion on follow-up, consistent with previous studies. . $^{\text {5-9,14 }}$ We also found no significant changes in the preprocedural and postprocedural parameters such as heart rate, blood pressure, respiratory rate, presence of rash, fever, urticaria, or chills after infusion of BMMNC. No other complication was seen in our study patients, which is consistent with previous similar studies. $^{5-9,14}$

The secondary end point in the present study was to evaluate clinical improvement based on the improvement in the modified Rankin Scale. Patients with a baseline NIHSS score of 8-14 ( $n=$ 17) did better than those with a baseline NIHSS score of $>14$ ( $n=$ 3 ). In the former group, 12 patients achieved good clinical outcome ( 8 in the intervention group and 4 in the control group; $P=$ .068 ). In the latter group, all 3 patients did not achieve the defined good clinical outcome of $\mathrm{mRS}<2$.

Friedrich et $\mathrm{al}^{9}$ also used similar criteria for good clinical outcome; however, their defined follow-up was 90 days, and there was no control group or randomization. They found good clinical outcome in $8(40 \%)$ of their patients as opposed to $80 \%$ in the 
present study. Moniche et $\mathrm{al}^{5}$ used controls as in our study and found that $20 \%$ of the patients who received therapy achieved an mRS of 2 at 6 months versus none in the control group; however, the number of patients showing improvement in mRS did not reach statistically significant levels $(P<.47)$.

We also found improving trends in the NIHSS, mRS, and BI in both our stem cell and control groups, which was statistically significant in the intervention group when comparing baseline to 6-month follow-up. Similar trends were also observed in the other studies. $^{5-9,14}$

There were several limitations of our study. The sample size in our study was small, thus limiting the power of this study to confidently claim clinical benefits in patients who received stem cells compared with controls. Patients with extremes of stroke severity as per the NIHSS were not equally represented due to small sample size; thus, definite conclusions about which patients will benefit the most cannot be made. Although all measures were taken to ensure blinding of the evaluating observers at 6 months, the lack of any sham procedure in the control group might have interfered with the efficacy of blinding.

\section{CONCLUSIONS}

The present study demonstrates the safety of intra-arterial BMMNC in patients with acute stroke, with a trend toward improved clinical outcome compared with control patients. Stem cells offer a promising novel therapy in these patients, with reduction in morbidity and improved functional outcome. Further randomized studies with a large patient cohort are needed to validate our findings.

\section{REFERENCES}

1. Intercollegiate Stroke Working Party. National Sentinel Stroke Clinical Audit 2010: Public Report for England, Wales and Northern Ireland. London: Royal College of Physicians; 2011. https://www. rcplondon.ac.uk/projects/outputs/national-sentinel-stroke-audit2010. Accessed on March 20, 2017

2. Powers WJ, Derdeyn CP, Biller J, et al; American Heart Association Stroke Council. 2015 Heart Association/American Stroke Association Focused Update of the $\mathbf{2 0 1 3}$ Guidelines for the early management of patients with acute ischemic stroke regarding endovascular treatment: a guideline for healthcare professionals from the Amer- ican Heart Association/American Stroke Association. Stroke 2015; 46:3024-35 CrossRef Medline

3. Morgenstern LB, Staub L, Chan W, et al. Improving delivery of acute stroke therapy: the TLL Temple Foundation Stroke Project. Stroke 2002;33:160-66 CrossRef Medline

4. Stroemer P, Patel S, Hope A, et al. The neural stem cell line CTX0E03 promotes behavioral recovery and endogenous neurogenesis after experimental stroke in a dose-dependent fashion. Neurorehabil Neural Repair 2009;23:895-909 CrossRef Medline

5. Moniche F, Gonzalez A, Gonzalez-Marcos JR, et al. Intra-arterial bone marrow mononuclear cells in ischemic stroke: a pilot clinical trial. Stroke 2012;43:2242-44 CrossRef Medline

6. Battistella V, de Freitas GR, da FonsecaLM, et al. Safety of autologous bone marrow mononuclear cell transplantation in patients with nonacute ischemic stroke. Regen Med 2011;6:45-52 CrossRef Medline

7. Suárez-Monteagudo C, Hernández-Ramırez P, Alvarez-González L, et al. Autologous bone marrow stem cell neurotransplantation in stroke patients: an open study. Restor Neurol Neurosci 2009;27: 151-61 Medline

8. Savitz SI, Misra V, Kasam M, et al. Intravenous autologous bone marrow mononuclear cells for ischemic stroke. Ann Neurol 2011;70: 59-69 CrossRef Medline

9. Friedrich MA, Martins MP, Araujo MD, et al. Intra-arterial infusion of autologous bone marrow mononuclear cells in patients with moderate to severe middle cerebral artery acute ischemic stroke. Cell Transplant 2012;21(suppl 1):S13-21 CrossRef Medline

10. National Institute of Neurological Disorders and Stroke rt-PA Stroke Study Group. Tissue plasminogen activator for acute ischemic stroke. N Engl J Med 1995;333:1581-87 CrossRef Medline

11. Jin K, Wang X, Xie L, et al. Evidence for stroke-induced neurogenesis in the human brain. Proc Natl Acad Sci U S A 2006;103:13198202 CrossRef Medline

12. Thomson JA, Itskovitz-Eldor J, Shapiro SS, et al. Embryonic stem cell lines derived from human blastocysts. Science 1998;282:1145-47 CrossRef Medline

13. Banerjee S, Williamson DA, Habib N, et al. The potential benefit of stem cell therapy after stroke: an update. Vasc Health Risk Manag 2012;8:569-80 CrossRef Medline

14. Banerjee S, Bentley P, Hamady M, et al. Intra-arterial immunoselected CD34+ stem cells for acute ischemic stroke. Stem Cells Transl Med 2014;3:1322-30 CrossRef Medline

15. Brenneman M, Sharma S, Harting M, et al. Autologous bone marrow mononuclear cells enhance recovery after acute ischemic stroke in young and middle-aged rats. J Cereb Blood Flow Metab 2010;30: 140-49 CrossRef Medline 